\title{
BMJ Open Efficacy and safety of ginkgo preparations for attention deficit hyperactivity disorder: a systematic review protocol
}

\author{
Sufei He, ${ }^{1}$ Miao Wang, ${ }^{2}$ Jinhua Si, ${ }^{3}$ Tianyi Zhang, ${ }^{4}$ Hong Cui, ${ }^{5}$ Xiumei Gao ${ }^{1}$
}

To cite: He S, Wang M, Si J, et al. Efficacy and safety of ginkgo preparations for attention deficit hyperactivity disorder: a systematic review protocol. BMJ Open 2018;8:e20434. doi:10.1136/ bmjopen-2017-020434

- Prepublication history for this paper is available online. To view these files, please visit the journal online (http://dx.doi. org/10.1136/bmjopen-2017020434).

SH and MW contributed equally.

Received 3 November 2017 Revised 20 December 2017 Accepted 25 January 2018

Check for updates

${ }^{1}$ Collaborative Innovation Center of Modern Chinese Medicine, Tianjin University of Traditional Chinese Medicine, Tianjin, China ${ }^{2}$ College of Chinese Medicine, Tianjin University of Traditional Chinese Medicine, Tianjin, China ${ }^{3}$ Department of Library, Tianjin University of Traditional Chinese Medicine, Tianjin, China ${ }^{4}$ Second Affiliated Hospital, Tianjin University of Traditional Chinese Medicine, Tianjin, China

${ }^{5}$ First Affiliated Hospital, Tianjin University of Traditional Chinese Medicine, Tianjin, China

Correspondence to

Professor Xiumei Gao; gaoxiumei1984@hotmail.com

\section{ABSTRACT}

Introduction Attention deficit hyperactivity disorder (ADHD) is one of the most commonly diagnosed and treated childhood psychiatric disorders. The analogous diagnosis adopted in Europe is hyperkinetic disorder, which is defined in the WHO's International Classification of Diseases 10th edition (ICD-10). Hyperkinetic disorder includes more severe conditions. Ginkgo preparations are used in the treatment of ADHD. The present study will assess the efficacy and safety of ginkgo preparations in the treatment of ADHD in the currently published literature. Materials and methods All prospective randomised controlled trials (RCTs) will be included in this systematic review. Patients diagnosed with $A D H D$ according to American Psychiatric Association's Diagnostic and Statistical Manual of Mental Disorders fourth edition (DSM-IV), Diagnostic and Statistical Manual of Mental Disorders, fifth edition (DSM-5), ICD-10 or Chinese Classification and Diagnosis of Mental Diseases third edition (CMDD) will be included. A comprehensive search for RCTs to evaluate the effectiveness and tolerance of ginkgo preparations will be performed. The primary outcomes are the ADHD Rating Scale-IV and Revised Conners' Parent Rating Scale. The secondary outcomes are quality of life evaluated by the KINDL scale, adverse effects/events, Conners' Teacher Rating Scale, Strengths and Weaknesses of ADHD Symptoms and Normal Behaviour Scale and Fremdbeurteilungsbogen für Hyperkinetische Störungen. Exclusion criteria are the following: (1) case reports, not randomised trial, non-comparative studies and (2) patients who were not diagnosed based on DSM-IV, DSM-5, ICD-10 or CMDD. The following databases will be searched from their inception until January 2018: Medline, Embase, the Cochrane Central Register of Controlled Trials, Web of Science, China Biology Medicine Disc, China National Knowledge Infrastructure Database, Wanfang Database and Chinese Scientific Journals Database. Two authors will independently perform the study selection, extract the data and assess the study quality and risk of bias.

Ethics and dissemination This systematic review does not require ethics approval. It will be published in a peerreviewed journal.

PROSPERO registration number CRD42017077190.

\section{INTRODUCTION}

Description of the condition of ADHD treatment The prevalence of attention deficit hyperactivity disorder (ADHD) in children and
Strengths and limitations of this study

- This study will evaluate the safety of ginkgo preparations as a sole or adjunct agent for attention deficit hyperactivity disorder (ADHD) treatment.

- Our review will be useful to clinicians, patients and parents who use ginkgo preparations for ADHD treatment.

- Clinical heterogeneity may exist for different dosage forms of ginkgo preparations, doses, durations and combined treatments.

- There may be a language bias with the limitation of English and Chinese studies.

adolescents is as high as $3.4 \%$ in the general population, ${ }^{1}$ and it is one of the most commonly diagnosed and treated childhood psychiatric disorders. ${ }^{2}$ The diagnosis rate rises to $6.26 \%$ in China. ${ }^{3}$ The analogous diagnosis adopted in Europe is hyperkinetic disorder, which is defined in the International Classification of Diseases 10th edition (ICD-10). Hyperkinetic disorder includes more severe conditions. ${ }^{4}$ ADHD is a childhood-onset neurodevelopmental disorder that may persist into adolescence and adulthood, and it has a high societal burden. The primary symptoms consist of inattention and hyperactivity/impulsivity that are often accompanied by other neurodevelopmental disorders, such as autism spectrum disorder ${ }^{5}$ and intellectual disability. ${ }^{6}$ A large proportion of adolescents and adults with ADHD exhibit antisocial behaviour and criminal activities, including conduct disorder, oppositional defiant disorder, ${ }^{7}$ risk of crashing, ${ }^{8}$ sexual offences $^{9}$ and arson, ${ }^{10}$ especially among arrested, convicted and imprisoned adolescents and adults. Therefore, ADHD has been increasingly considered a severe social issue.

Stimulants are the first-line medications for ADHD treatment. Patients with ADHD manage their symptoms by using stimulants. 
However, the risk of substance abuse may increase in this patient population, and substance use disorder is one of the most common comorbid psychiatric disorders in adolescent and adult patients. ${ }^{11} 12$ The related adverse side effects of stimulants include cardiovascular events, insomnia, appetite loss, hypoevolutism, gastrointestinal symptoms and tics. ${ }^{13}$ Complementary or alternative medical treatments for ADHD, such as plant-based medications, acupuncture ${ }^{14}$ and music therapy, ${ }^{15}$ are considered because of the side effects, abuse and misuse of conventional pharmacological treatments. It is also important to evaluate the efficacy and safety of plantbased medications and acupuncture.

\section{Description of the intervention}

Ginkgo biloba preparations, including tablets, granules, pills, injection distillates, oral solutions, extracts and dropping pills, are approved for commercial marketing. Egb 761, Ginaton, Tebonin, Rokan, Tanakan, Ginkobil, GBE50 and Kaveri are approved in the USA and Europe. Ginkgo Biloba Leaves Dispersible Tablet, Ginkgo Leaf Capsule, Ginkgo Leaves Soft Gel Capsule, Ginkgo Damole Injection, Yinxing Damo, Ginkgo Biloba Granule, Yinxing Guttate Dropping Pill, Ginkgo Biloba Extract Injection, Ginkgo Distillate, Diterpene Ginkgolides Meglumine Injection, Ginkgolide Injection, Ginkgo Biloba Leaves Extract Oral Solution, Ginkgo Leaf Extract, Armillariella Mellea Powders Oral Solution, Yinxing Guttate Dropping Pills and Shuxuening Zhusheye are approved by the China Food and Drug Administration. Ginkgo preparations are among the bestselling botanical dietary supplements worldwide. Clinical evidence indicates that Ginkgo biloba is safe and exhibits no excess side effects compared with placebo for cognitive impairment and dementia. ${ }^{16}$ However, the evidence of efficacy is equivocal. ${ }^{17}$

Ginkgo preparations alleviate the conditions such as autism, ${ }^{18}$ depression ${ }^{19}$ and neuropsychiatric symptoms such as anxiety. ${ }^{20}$ Ginkgo preparations may affect the behavioural and cognitive aspects of ADHD. The predominant behavioural effects are calming and improved frustration tolerance. Ginkgo biloba induces willful cognition, discriminant attention and decreases irritability. ${ }^{21}$

\section{How the intervention might work}

Components isolated from ginkgo biloba contain terpene trilactones, flavonol glycosides, isoflavonoids, biflavones, proanthocyanidins, alkylphenols, carboxylic acids, 4-O-methylpyridoxine and polyprenols. ${ }^{22-24}$ Preclinical evidence indicates that ginkgo flavonol glycosides are predominantly responsible for the antioxidant activity. ${ }^{25}$ The antioxidant activity of ginkgo flavonol glycosides reduce reactive oxygen species-induced oxidative stress, which contributes to neurodevelopmental disorders by causing membrane damage, changes in protein structure and function, lipid denaturation and DNA damage. ${ }^{26}$ Terpene trilactones are associated with neuroprotective properties. ${ }^{27}$ Investigations have demonstrated that terpene trilactones attenuate the decrease in brain-derived neurotropic factor, norepinephrine transporter and dopamine transporter, ${ }^{28}$ which are negatively related to ADHD pathogenesis. ${ }^{29-31}$

No comprehensive quantitative reviews of treatments of ADHD with ginkgo preparations have been performed. Therefore, it is important to investigate the current evidence of the efficacy and tolerance of ginkgo preparations therapy for ADHD.

\section{MATERIALS AND METHODS}

\section{Registration information}

This systematic review protocol adheres to the preferred reporting items for systematic review and meta-analysis protocols (PRISMA-P) 2015. ${ }^{32}$ The protocol was registered on PROSPERO, and the registration number is CRD42017077190.

\section{Inclusion criteria}

Types of study

All prospective randomised controlled trials (RCTs) will be included in this systematic review, but trials without detailed data will be excluded. Abstracts with sufficient outcome data will be included. Cross-over trials will be included with the two phases of data if there is sufficient washout and return to baseline.

\section{Type of participants}

Patients with ADHD or hyperkinetic disorder who were diagnosed based on American Psychiatric Association's Diagnostic and Statistical Manual of Mental Disorders fourth edition (DSM-IV), Diagnostic and Statistical Manual of Mental Disorders fifth edition (DSM-5), WHO's International Classification of Diseases 10th edition (ICD10) or Chinese Classification and Diagnosis of Mental Diseases third edition (CMDD) will be included. There will be no limitations on age or sex.

\section{Types of interventions and controls}

Randomised studies of the ginkgo preparations, as the sole treatment or as an adjunct to other treatments, which were used in the intervention and control groups in the same manner will be included. Ginkgo preparations include Egb 761, Ginaton, Tebonin, Rokan, Tanakan, Ginkobil, GBE50 and Kaveri, Ginkgo Biloba Leaves Dispersible Tablet, Ginkgo Leaf Capsule, Ginkgo Leaves Soft Gel Capsule, Ginkgo Damole Injection, Yinxing Damo, Ginkgo Biloba Granule, Yinxing Guttate Dropping Pill, Ginkgo Biloba Extract Injection, Ginkgo Distillate, Diterpene Ginkgolides Meglumine Injection, Ginkgolide Injection, Ginkgo Biloba Leaves Extract Oral Solution, Ginkgo Leaf Extract, Armillariella Mellea Powders Oral Solution, Yinxing Guttate Dropping Pills and Shuxuening Zhusheye. The control groups will include psychostimulant drug use, placebo use, psychotherapy and no treatment. 


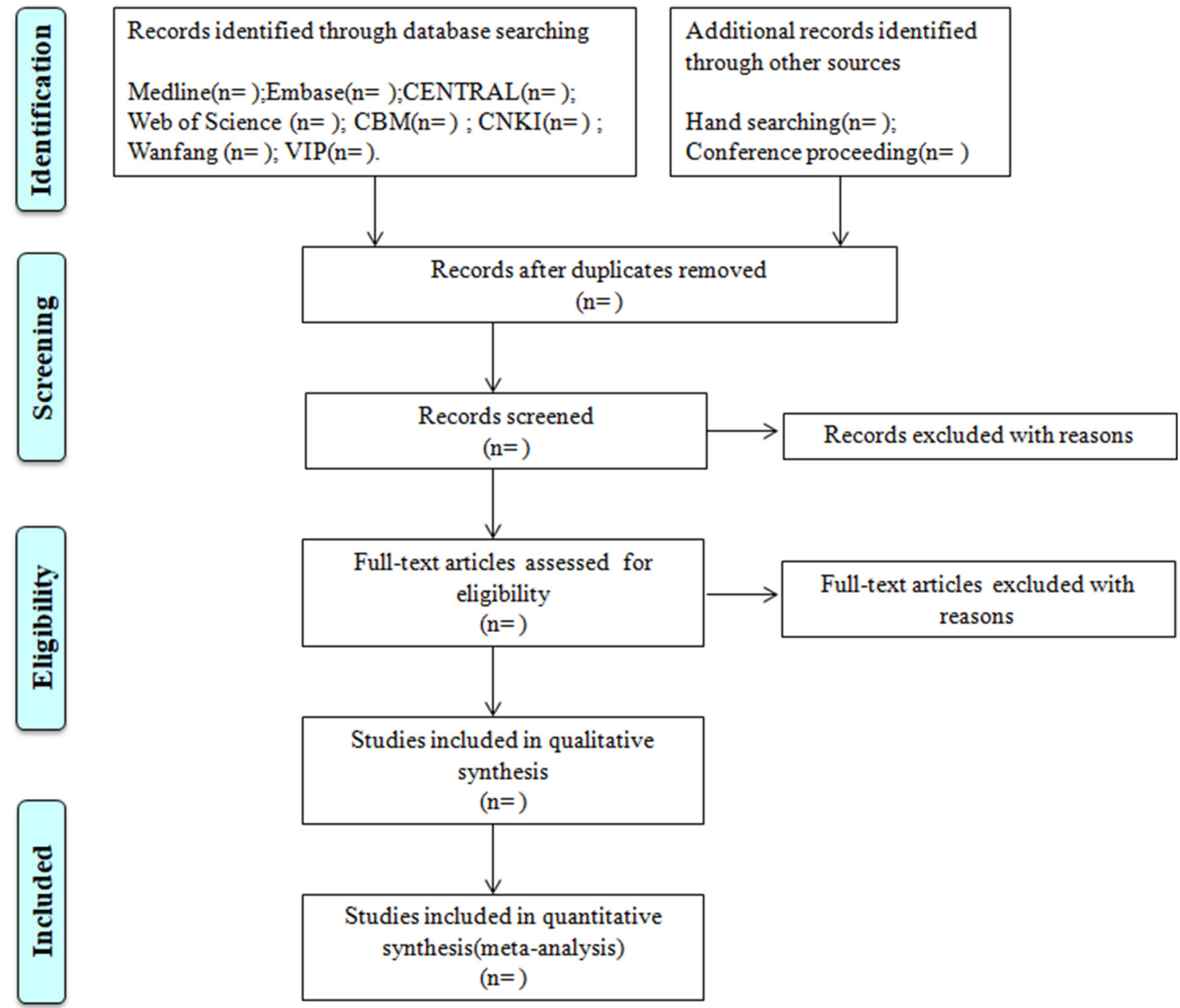

Figure 1 Preferred reporting items for systematic review and meta-analysis flow diagram of the study searching and screening.

\section{Outcomes measures}

Primary outcomes

The ADHD Rating Scale-IV (ADHD-RS-IV).

The Revised Conners' Parent Rating Scale (CPRS-R). ${ }^{33}$

Secondary outcomes

Quality of life on the KINDL scale. ${ }^{34}$

Adverse effects/events.

Conners' Teacher Rating Scale (CTRS). ${ }^{35}$

Strengths and Weaknesses of ADHD Symptoms and Normal Behaviour (SWAN) Scale. ${ }^{36}$

Fremdbeurteilungsbogen für

Störungen (FBB-HKS) ${ }^{37}$

\section{Exclusion criteria}

1. Case reports, not randomised trial, non-comparative studies.

2. Patients who were not diagnosed based on DSM-IV, DSM-5, ICD-10 or CMDD.

\section{Data sources}

We will search the following electronic bibliographic databases: Medline, Embase, the Cochrane Central Register of Controlled Trials (CENTRAL), Web of Science (science and social science citation index), China Biology Medicine Disc (CBMdisc), China National Knowledge Infrastructure Database (CNKI), Wanfang Database and the Chinese Scientific Journals Database (VIP). The search strategy will include terms relating to or describing the patients and intervention. The terms will be combined with the Cochrane MEDLINE filter for controlled trials of interventions.

\section{Search strategies}

A search of Medline will be performed using the following terms: (ginkgo biloba OR ginkgolides OR bilobalides OR ginkgo biloba leaves dispersible tablet OR Ginkgo Leaf Capsule OR Ginkgo Leaves Soft gel Capsule OR Ginkgo damole Injection OR Yinxing damo OR Ginkgo biloba granule OR Yinxing Guttate Dropping Pill OR Ginkgo biloba extract injection OR ginkgo distillate OR Diterpene Ginkgolides Meglumine Injection OR Ginkgolide Injection OR Ginkgo Biloba Leaves Extract Oral Solution OR Ginkgo Leaf Extract OR Armillariella Mellea Powders Oral Solution OR Yinxing Guttate Dropping Pills OR Egb 761 OR Egb761 OR Ginaton OR Tebonin forte OR Tanakan OR Rökan OR Ginkobil OR GBE50 OR Kaveri 
OR Shuxuening Zhusheye) AND (attention deficit hyperactivity disorder OR ADHD OR hyperkinetic disorders). The strategies will be modified for Embase, Cochrane, Web of Science, CBM, CNKI, Wanfang and VIP.

The search terms will be adapted for use with the above bibliographic databases in combination with database-specific filters for controlled trials, when available. Language is limited with English and Chinese. The databases will be searched from their inception until January 2018.

\section{Data collection and analysis}

Selection of studies

Two authors ( $\mathrm{SH}$ and MW) will select studies by assessing the titles and abstracts after duplication removal. The full text will be further reviewed for inclusion. RCTs that investigated the efficacy and safety of medication therapy in patients diagnosed with ADHD will be selected. Study selection will be documented and summarised in a PRISMA-compliant flow chart (figure 1) (http://www.prismastatement.org).

\section{Data extraction}

Two authors (SH and $\mathrm{HC}$ ) will independently perform data extraction and risk of bias assessments using a predefined data extraction form. The first author, publication year, study design, intervention, dosage, diagnostic criteria, disease duration, number of participants allocation, dropout, duration, outcome, outcome results (eg, ADHD-RS-IV, CPRS-R, quality of life on the KINDL scale and adverse effects/events) and follow-up periods will be collected. We will use GRADEpro software to create a summary of findings table.

Any disagreement between the two authors will be resolved by discussion, and XG will make the final decision. TZ will contact the authors for additional information by mail when the data are insufficient.

\section{Assessment of study quality and risk of bias}

The quality of the studies for each outcome will be assessed using the Grading of Recommendations Assessment, Development and Evaluation classification system, which will be judged by limitations in the design and implementation, imprecision, inconsistency, indirectness and reporting bias. Evidence quality will be classified into four levels: high, moderate, low or very low.

We will assess the risk of bias of the included studies using a risk of bias assessment tool according to the guidelines of the Cochrane Handbook. ${ }^{38} \mathrm{SH}$ and MW will classify the risk of bias in the included studies as low risk, unclear risk and high risk. The following factors will be assessed: random sequence generation, allocation sequence concealment, blinding of participants and personnel, blinding of outcome assessment, incomplete outcome data and selective outcome reporting.

\section{Data synthesis and analysis}

A meta-analysis will be performed using a random-effect model or fixed-effect model for the identified studies. We will use Review Manager V.5.3.5 software (RevMan
V.5.3.5) to combine the data from the trials. The mean differences or standardised mean differences for continuous outcomes and risk ratio for dichotomous outcomes with 95\% CIs will be pooled in RevMan V.5.3.5. A systematic narrative synthesis will be performed to summarise the relationship of the included studies when quantitative synthesis is not appropriate.

\section{Dealing with missing data}

We will attempt to collect data from original study investigators if possible for missing or incomplete data. We will consider estimating data if we cannot obtain the original source.

\section{Assessment of reporting biases}

We will detect reporting biases using funnel plots if more than 10 studies are included. An asymmetry test will be performed using Egger's method. ${ }^{39}$

\section{Assessment of heterogeneity}

Heterogeneity will be tested using the $\chi^{2}$ test for the $\mathrm{P}$ value. $\mathrm{I}^{2}$ will be calculated. An $\mathrm{I}^{2}$ value $>50 \%$ will indicate substantial heterogeneity. ${ }^{40}$ We will perform subgroup analyses or narrative descriptions based on the situation (eg, lack of included trials).

\section{Subgroup analysis and investigation of heterogeneity}

Subgroup analysis will be performed to explore the source of heterogeneity by the different dosage forms of ginkgo preparations, dose, follow-up period and type of control.

\section{Sensitivity analysis}

Low-quality trials will be excluded from sensitivity analyses according to the different effect models. A summary table will report the results of the sensitivity analyses.

\section{DISCUSSION}

Our systematic review will provide a detailed summary of the efficacy and tolerance of ginkgo preparations for the treatment of ADHD. Ginkgo preparations are used in ADHD treatment, but no systematic reviews on the efficacy or safety have been published. We will identify ginkgo preparation subtypes that are particularly useful in specific subgroups. We hope that our study will provide a reference for physicians, patients and parents in ADHD clinical practice.

Contributors SH, MW and XG drafted the protocol. SH and JS developed and will perform the search strategy. SH and MW will select the studies. SH and HC will extract data from selected studies. SH and TZ will perform the analyses. All of the authors read and approved the final protocol. SH and MW will update this review.

Funding This study is supported by the National Natural Science Foundation of China (No. 81630106).

Competing interests None declared.

Patient consent Not required.

Ethics approval This systematic review does not require ethics approval. It will be published in a peer-reviewed journal.

Provenance and peer review Not commissioned; externally peer reviewed. 
Open Access This is an Open Access article distributed in accordance with the Creative Commons Attribution Non Commercial (CC BY-NC 4.0) license, which permits others to distribute, remix, adapt, build upon this work non-commercially, and license their derivative works on different terms, provided the original work is properly cited and the use is non-commercial. See: http://creativecommons.org/ licenses/by-nc/4.0/

(c) Article author(s) (or their employer(s) unless otherwise stated in the text of the article) 2018. All rights reserved. No commercial use is permitted unless otherwise expressly granted.

\section{REFERENCES}

1. Polanczyk GV, Salum GA, Sugaya LS, et al. Annual research review: a meta-analysis of the worldwide prevalence of mental disorders in children and adolescents. J Child Psychol Psychiatry 2015;56:345-65

2. Scahill L, Schwab-Stone M. Epidemiology of ADHD in school-age children. Child Adolesc Psychiatr Clin N Am 2000;9:541-55.

3. Wang T, Liu K, Li Z, et al. Prevalence of attention deficit/hyperactivity disorder among children and adolescents in China: a systematic review and meta-analysis. BMC Psychiatry 2017;17:1-11.

4. Ford T, Goodman R, Meltzer H. The British child and adolescent mental health survey 1999: the prevalence of DSM-IV disorders. J Am Acad Child Adolesc Psychiatry 2003;42:1203-11.

5. Antshel KM, Zhang-James Y, Faraone SV. The comorbidity of ADHD and autism spectrum disorder. Expert Rev Neurother 2013;13:1117-28.

6. Faraone SV, Ghirardi L, Kuja-Halkola R, et al. The familial coaggregation of attention-deficit/hyperactivity disorder and intellectual disability: a register-based family study. J Am Acad Child Adolesc Psychiatry 2017:56:167-74.

7. Langley K, Fowler T, Ford T, et al. Adolescent clinical outcomes for young people with attention-deficit hyperactivity disorder. $\mathrm{Br} \mathrm{J}$ Psychiatry 2010;196:235-40.

8. Curry AE, Metzger KB, Pfeiffer MR, et al. Motor vehicle crash risk among adolescents and young adults with attention-deficit/ hyperactivity disorder. JAMA Pediatr 2017;171:756-63.

9. Kafka M. Axis I psychiatric disorders, paraphilic sexual offending and implications for pharmacological treatment. Isr J Psychiatry Relat Sci 2012;49:255-61.

10. Lambie I, loane J, Randell I, et al. Offending behaviours of child and adolescent firesetters over a 10-year follow-up. J Child Psychol Psychiatry 2013;54:1295-307.

11. Martinez-Raga J, Knecht C, de Alvaro R, et al. Addressing dual diagnosis patients suffering from attention-deficit hyperactivity disorders and comorbid substance use disorders. Addict Disord Their Treat 2013;12:213-30.

12. Norén Selinus E, Molero Y, Lichtenstein P, et al. Subthreshold and threshold attention deficit hyperactivity disorder symptoms in childhood: psychosocial outcomes in adolescence in boys and girls. Acta Psychiatr Scand 2016;134:533-45.

13. Thapar A, Cooper M. Attention deficit hyperactivity disorder. The Lancet 2016;387:1240-50.

14. Molsberger F, Raak C, Witthinrich C. Improvements in sleep and handwriting after complementary medical intervention using acupuncture, applied kinesiology, and respiratory exercises in a nine-year-old ADHD patient on methylphenidate. Explore 2014;10:398-403.

15. Rothmann K, Hillmer JM, Hosser D. [Evaluation of the musical concentration training with pepe (MusiKo mit Pepe) for children with attention deficits]. Z Kinder Jugendpsychiatr Psychother 2014;42:325-35

16. Birks J, Grimley Evans J. Ginkgo biloba for cognitive impairment and dementia. Cochrane Database Syst Rev 2009;36:CD003120.

17. Tan MS, Yu JT, Tan CC, et al. Efficacy and adverse effects of ginkgo biloba for cognitive impairment and dementia: a systematic review and meta-analysis. J Alzheimers Dis 2015;43:589-603.

18. Solomon PR, Adams F, Silver A, et al. Ginkgo for memory enhancement: a randomized controlled trial. JAMA 2002;288:835-40.

19. Hasanzadeh E, Mohammadi M-R, Ghanizadeh A, et al. A doubleblind placebo controlled trial of ginkgo biloba added to risperidone in patients with autistic disorders. Child Psychiatry Hum Dev 2012;43:674-82.
20. Gavrilova SI, Preuss UW, Wong JWM, et al. Efficacy and safety of Ginkgo biloba extract EGb 761 in mild cognitive impairment with neuropsychiatric symptoms: a randomized, placebocontrolled, double-blind, multi-center trial. Int J Geriatr Psychiatry 2014;29:1087-95.

21. Niederhofer H. Ginkgo biloba treating patients with attention-deficit disorder. Phytother Res 2010;24:26-7.

22. van Beek TA, Montoro P. Chemical analysis and quality control of Ginkgo biloba leaves, extracts, and phytopharmaceuticals. J Chromatogr A 2009;1216:2002-32.

23. Li L, Zhao Y, Du F, et al. Intestinal absorption and presystemic elimination of various chemical constituents present in GBE50 extract, a standardized extract of Ginkgo biloba leaves. Curr Drug Metab 2012;13:494-509.

24. Cao J, Yang M, Cao F, et al. Tailor-made hydrophobic deep eutectic solvents for cleaner extraction of polyprenyl acetates from Ginkgo biloba leaves. J Clean Prod 2017;152:399-405.

25. Pilija V, Mirjana R, Brenesel MD, et al. Inhibitory Effect of ginkgo biloba extract on the tonus of the small intestine and the colon of rabbits. Molecules 2010;15:2079-86.

26. Popa-Wagner A, Mitran S, Sivanesan S, et al. ROS and brain diseases: the good, the bad, and the ugly. Oxid Med Cell Longev 2013;2013:1-14

27. Singh S, Barreto G, Aliev G, et al. Ginkgo biloba as an alternative medicine in the treatment of anxiety in dementia and other psychiatric disorders Curr Drug Metab 2017:18:112-9.

28. Nam Y, Shin E-J, Shin SW, et al. YY162 prevents ADHD-like behavioral side effects and cytotoxicity induced by Aroclor1254 via interactive signaling between antioxidant potential, BDNF/TrkB, DAT and NET. Food ChemToxicol 2014;65:280-92.

29. Corominas-Roso M, Ramos-Quiroga JA, Ribases M, et al. Decreased serum levels of brain-derived neurotrophic factor in adults with attention-deficit hyperactivity disorder. Int $J$ Neuropsychopharmacol 2013;16:1267-75.

30. Fentress HM, Klar R, Krueger JJ, et al. Norepinephrine transporter heterozygous knockout mice exhibit altered transport and behavior Genes Brain Behav 2013;12:749-59.

31. Mereu M, Contarini G, Buonaguro EF, et al. Dopamine transporter (DAT) genetic hypofunction in mice produces alterations consistent with ADHD but not schizophrenia or bipolar disorder. Neuropharmacology 2017;121:179-94.

32. Shamseer $L$, Moher $D$, Clarke $M$, et al. Preferred reporting items for systematic review and meta-analysis protocols (PRISMA-P) 2015 elaboration and explanation. BMJ 2015;349:97647.

33. Conners CK, Sitarenios G, Parker JD, et al. The revised Conners Parent Rating Scale (CPRS-R): factor structure, reliability, and criterion validity. J Abnorm Child Psychol 1998;26:257-68.

34. Ravens-Sieberer U, Bullinger M. Assessing health-related quality of life in chronically ill children with the German KINDL: first psychometric and content analytical results. Qual Life Res 1998;7:399-407.

35. Conners CK, Sitarenios G, Parker JD, et al. Revision and restandardization of the Conners Teacher Rating Scale (CTRS-R): factor structure, reliability, and criterion validity. J Abnorm Child Psychol 1998;26:279-91.

36. Lai KYC, Leung PWL, Luk ESL, et al. Validation of the Chinese strengths and weaknesses of ADHD-symptoms and normalbehaviors questionnaire in Hong Kong. J Atten Disord 2013;17:194-202.

37. Brühl B, Döpfner M, Lehmkuhl G. Der fremdbeurteilungsbogen für hyperkinetische störungen (FBB-HKS) - prävalenz hyperkinetischer störungen im elternurteil und psychometrische kriterien. Kindheit und Entwicklung 2000;9:116-26.

38. Higgins JPT, Altman DG, Sterne JAC. Chapter 8: assessing risk of bias in included studies. In: Higgins JPT, Green S, eds. Cochrane handbook for systematic reviews of interventions version 5.1.0, 2011 (updated March 2011): The Cochrane Collaboration, 2011.

39. Egger M, Smith GD, Schneider M, et al. Bias in meta-analysis detected by a simple, graphical test. BMJ 1997;315:629-34.

40. Higgins JPT, Altman DG. The Cochrane Collaboration. Chapter 9: Analysing data and undertaking meta-analyses. In: Higgins JPT, Green S, eds. Cochrane handbook for systematic reviews of interventions version 5.1.0, 2011 (updated March 2011): The Cochrane Collaboration, 2011. 